\title{
HOMMAGE TO DR. NORMAN E. BORLAUG
}

Dr. Norman E. Borlaug, the distinguished agriculture researcher and 1970 Nobel Peace Prize, died in Texas, United States on September 12, 2009.

$\mathrm{He}$ is considered to be the father of the Green Revolution in the world and the central axis of his work was in the Centro Internacional de Mejoramiento de Maíz y Trigo (CIMMYT) located in Mexico. From there, he extended hundreds of technological networks with dozens of countries, especially with those of the developing world, though the doors of his big field laboratory were open to scientists from the whole world without distinction. These networks brought knowledge along with the improved wheat varieties that replaced with astonishing success the old seeds that some peasants had sown for generations.

One of the most important aspects of his great work in the field of wheat breeding was the creation of dwarf or short varieties resistant to disease, high yield grain, and above all, largely adaptable. He made it possible for his new varieties to be distributed to diverse parts of the world where they contributed in mitigating the hunger of millions of people, and as a result, received a welldeserved Nobel Peace Prize in 1970.

Many countries, among them Chile, benefited with the wheat genetic material created by this scientist who incarnated the concept that it is better to teach how to fish than giving away fish. His intellectual openness and interest to impart knowledge surpassed every limit and he shared with researchers in the wheat fields of the CIMMYT in Obregón, Mexico, up until a few months before his death, unconcerned about his advanced age and failing health. It is probable that some people who were present during his last visit to those breeding nurseries will have thought that his work as a plant breeder, started in 1945 in Valle del Yaqui (Mexico) by candlelight, over the years would be transmitted throughout the world as if it were the greatest spark that nature can produce.

His conferences and meetings in the field, whether for a very specialized or less learned public, had a very educational and stimulating content, but above all, an enviable simplicity. Justifying this teaching style, he pointed out that the person who understands what $\mathrm{s} / \mathrm{he}$ says will always find a simple means to explain it. In this way, many of the genetic and statistical concepts led him to terminology that could be assimilated even by the most humble peasant. He related that when he wanted to demonstrate that a variety of wheat was superior to another, or that a certain dose of fertilizer was the best, instead of talking about "significant differences", it was enough for him to gather together the harvest of each treatment so that the farmer could observe it, compare it, and come to his own conclusions. In spite of being an elite scientist, his great modesty and quality as a person led him with an absolutely natural manner to interact and treat others as equals, demonstrating great generosity, especially with the most humble peasants of Asia, Africa, or Latin America whom he remembered a great deal in his conferences and in informal conversations.

We were privileged that on various occasions he visited Chile with the aim of sharing his knowledge and experiences with scientists connected to wheat breeding and agronomy, as well as with the government authorities at that time.

In the Instituto de Investigaciones Agropecuarias (INIA), he shared his experiences from around the world with plant breeders, phytopathologists, and wheat specialists, among them René Cortázar S., Ignacio Ramírez A., Patricio Parodi P., Lilián Aguayo Ch., Juan Acevedo A., Cristián Hewstone M., Oscar Moreno M., Rodolfo Gonzales B., Héctor Wulf M., Denise Granger Z., Milan Caglevic C., Ernesto Hacke E., Ricardo Madariaga B., Iván Matus T., Claudio Jobet F., and the undersigned. I can point out with absolute certainty that the above-mentioned, some of whom have already left us, were very fortunate to know and share knowledge and experiences with Dr. Borlaug.

Without a doubt, there will be many countries that will pay tribute to this great agronomist researcher who is and will be an example of life and science for all those who have embraced the area of creating knowledge.

With profound admiration

\section{Mario Mellado Z.}

Wheat breeder 


\section{HOMENAJE AL DR. NORMAN E. BORLAUG}

El 12 de septiembre de 2009 falleció en Estados Unidos, Texas, el insigne investigador agrícola y premio Nobel de la Paz 1970, Dr. Norman E. Borlaug.

Es considerado el padre de la Revolución Verde en el mundo y el eje matriz de su trabajo estuvo radicado en el Centro Internacional de Mejoramiento de Maíz y Trigo (CIMMYT) ubicado en México. Desde ahí extendió cientos de redes tecnológicas con decenas de países, especialmente con aquellos del mundo en desarrollo, aunque las puertas de su gran laboratorio de campo estaban abiertas a los científicos de todo el orbe sin distinción de ningún tipo. Esas redes llevaban el conocimiento junto a las variedades mejoradas de trigo que reemplazaban con un éxito asombroso a las antiguas semillas que algunos campesinos habían sembrado por generaciones.

Uno de los aspectos más importantes de su magna obra en el ámbito del mejoramiento genético de trigo fue la creación de variedades enanas o de baja altura, con resistencia a enfermedades, de elevado rendimiento de grano y, sobre todo, de amplia adaptación. Ello hizo posible que sus nuevas variedades se distribuyeran por diversas latitudes del mundo, donde contribuyeron a mitigar el hambre de millones de gentes, y a raíz de ello recibió su merecido Premio Nobel de la Paz el año 1970.

Muchos países, entre ellos Chile, se beneficiaron con el material genético de trigo creado por este científico que hizo carne el concepto que más vale enseñar a pescar que regalar los peces. Su apertura intelectual e interés por entregar conocimiento sobrepasó todo límite y es así como estuvo compartiendo con investigadores en los campos de trigo del CIMMYT en Obregón, México, sólo hasta unos pocos meses antes de fallecer, sin importarle su avanzada edad y su delicado estado de salud. Es probable que algunas de las personas presentes en su última visita a esos viveros de mejoramiento hayan pensado que su trabajo de fitomejorador, que inició el año 1945 en el Valle del Yaqui (México) a la luz de una vela, con el correr de los años se irradió por el mundo como si fuese el mayor destello que la naturaleza puede producir.

Sus conferencias y reuniones de campo, fuesen para un público muy especializado o para otro menos letrado, eran de un contenido muy educativo y estimulante, pero sobre todo de una sencillez envidiable. Justificando esta manera de enseñar señalaba que la persona que entiende lo que dice siempre encontrará una forma sencilla de explicarlo. De esta manera, muchos de los conceptos de genética y de estadística los llevaba a una terminología que podía ser asimilada hasta por el campesino más humilde. Relataba que cuando él quería demostrar que una variedad de trigo era superior a otra, o que cierta dosis de fertilizante era la mejor, en vez de hablar de "diferencias significativas", le bastaba con juntar la cosecha de cada tratamiento para que el agricultor observara, comparara, y obtuviera sus propias conclusiones. Su gran modestia y calidad humana, a pesar de ser un científico de elite, lo conducía con absoluta naturalidad a interactuar y tratar a todos por igual, demostrando una gran generosidad, especialmente con los más humildes campesinos de Asia, África o América Latina, a quienes tanto recordaba en sus conferencias, así como en conversaciones informales.

Tuvimos el privilegio que visitara en varias oportunidades Chile, con el fin de compartir sus conocimientos y experiencias con científicos relacionados con mejoramiento y agronomía del trigo, así como con autoridades de gobierno de la época.

En el Instituto de Investigaciones Agropecuarias (INIA) repartió sus vivencias obtenidas por el mundo, con los fitomejoradores, fitopatólogos y especialistas en trigo, entre ellos René Cortázar S., Ignacio Ramírez A., Patricio Parodi P., Lilián Aguayo Ch., Juan Acevedo A., Cristián Hewstone M., Oscar Moreno M., Rodolfo Gonzales B., Héctor Wulf M., Denise Granger Z., Milan Caglevic C., Ernesto Hacke E., Ricardo Madariaga B., Iván Matus T., Claudio Jobet F., y el suscrito. Puedo señalar con absoluta certeza que todos los mencionados, algunos de los cuales ya no nos acompañan, fuimos muy afortunados al conocer y compartir conocimientos y experiencias con el Dr. Borlaug.

Sin duda que serán muchos países los que rendirán tributo a este gran agrónomo investigador que es y será un ejemplo de vida y de ciencia para todos quienes hayan abrazado el área de la creación de conocimiento.

Con profunda admiración

Mario Mellado Z.

Fitomejorador de trigo

Investigador emérito

Instituto de Investigaciones Agropecuarias INIA 